\title{
Direct and Indirect Effects of Janani Suraksha Yojana on Antenatal Care and Institutional Delivery
}

Mohammad Mahbubur Rahman ${ }^{1 *}$, and Saseendran Pallikadavath ${ }^{1}$.

${ }^{1}$ School of Health Sciences and Social Works, University of Portsmouth, UK.

*Corresponding Author : Mohammad Mahbubur Rahman, Department Portsmouth-Brawijaya Centre for Global Health, Population. UK. E-mail: mahbubur72@hotmail.com

Received date: October 24, 2018;Accepted date : February 01, 2018; Published date: February 27, 2019.

Citation : Mohammad Mahbubur Rahman, and Saseendran Pallikadavath. Direct and Indirect Effects of Janani Suraksha Yojana on Antenatal Care and Institutional Delivery.Women Health Care And Issues. Doi:10.31579/2642-9756/ 006

Copyright : (c) 2019 Mohammad Mahbubur Rahman.This is an open-access article distributed under the terms of The Creative Commons Attribution License, whichpermits unrestricted use, distribution, and reproduction in any medium, provided the original author and source are credited.

\section{Abstract}

Janani Suraksha Yojana (JSY) provides conditional cash incentives to eligible pregnant women in India so that they can take at least three antenatal care services including tetanus injection and iron-folic acid, to deliver their babies in health institutions, and to take at least one postnatal service for them and their neonatal babies. We examine its effects on antenatal care and the institutional delivery. We also decompose effects into direct and indirect categories, deriving parameters from the theoretical model, then estimating them using several regression methods including the propensity score matching. We use the fourth round of the District Level Household Survey (DLHS), which has an advantage over the previous waves of DLHS.

Key words: Janani Suraksha Yojana; demand-side financing, propensity score matching

\section{Introduction}

Every year globally, on average 303,000 women die at the time of pregnancy and childbirth, around 2.7 million babies die within the first 28 days of their life, and stillbirths are approximately 2.6 million (WHO, 2016). Pregnant women's lack of access to quality skilled care before, during and after childbirth, is identified as the main reason for these deaths (WHO, 2017). If uptakes of maternal and child health care services increase, these deaths are expected to reduce significantly.

For many years, by increasing facilities of maternal and child health care services, policymakers were trying to reduce maternal and child mortality. However, day by day, they also realized that an increase in the supply of healthcare facilities, such as medical staff, medicines, and machines, alone cannot increase uptakes of maternal and child health care services at an expected level, especially in a setting where health care facilities are not free, and prices of such facilities are not attainable for more impoverished people (Bhatia et al., 2006; Bhatia and Gorter, 2007; Ensor and Cooper, 2004; Koblinsky et al., 2006; Thaddeus and Maine, 1994).

The success of conditional cash transfer programs (for example, Progressa in Mexico), in the case of human capital and general health in the 1990s, motivated many policymakers of public health. In the spirit of Progressa, since the late 1990s, many developing countries have started conditional cash transfer programs to increase the uptakes of maternal and child health care services (Ensor et al., 2017; Yang et al., 2016; Kuwawenaruwa et al., 2016; Kingkaew et al., 2016; Engineer et al., 2016; Skiles et al., 2015). Such programs are commonly called Demand Side Financing (DSF) program, as they are meant to increase the demand for maternal and child health care services by reducing the relative prices of them (Gertler and Van der Gaag, 1990). Besides the supply side improvement policy, a DSF program has been found useful in increasing the uptakes of desired maternal and child health care services in most of the cases (Anwar et al., 2008, Behrman and Knowles, 1998, Bhatia et al., 2006, Bhatia and Gorter, 2007, Van de Poel et al., 2014).

In the context of high rates of maternal and neonatal death, India launched the world's largest DSF program, named as Janani Suraksha Yojana (JSY or safe motherhood scheme in English), in 2005.

Auctores Publishing - Volume1-004 www.auctoresonline.org Page - 01
The ultimate goal of JSY is to reduce maternal and neonatal death through increasing the institutional delivery. It covers more than 10 million women a year (MoHFW, 2017) and provides conditional cash transfers to eligible pregnant women so that they can give birth in public or accredited private healthcare facilities, they receive at least three antenatal care (ANC) services including tetanus (TT) injection and iron-folic acid (IFA), and at least one postnatal care (PNC) service for them and their neonatal babies (Yojana, 2006).

A number of studies have previously discussed JSY's effects on intended and unintended maternal and child health care outcomes (Nandi and Laxminarayan, 2016; Powell-Jackson et al., 2015; Carvalho et al., 2014; Gopalan and Durairaj, 2012; Gupta et al., 2011; Gupta et al., 2012; Lim et al., 2010; Modugu et al., 2012). All of these studies, which focused on institutional delivery, have established high positive effects of JSY on institutional delivery. However, findings of the effects of JSY on ANC and PNC are varied, as statistically insignificant effects have also been found on these intended outcomes (Powell-Jackson et al., 2015). On the other hand, significant effects have been found on some unintended outcomes (Powell-Jackson et al., 2015). For example, JSY significantly increases the probability of pregnancy, and JSY women start breastfeeding their neonatal babies earlier than non-JSY women.

Previous prominent studies (Nandi and Laxminarayan, 2016; PowellJackson et al., 2015; Carvalho et al., 2014; Lim et al., 2010) used data from second and third rounds of a repeated cross-section, District Level Household Survey (DLHS), surveyed in 2002-2004 and 2007-2008 respectively. The second round was surveyed before JSY, and the third round was surveyed when JSY was in its early stages when many women were not aware of it. There is a potential for the selection bias in that early round. The chance is high because JSY's operation has been running since 2007 (Das et al., 2011). Additionally, many women responded as JSY beneficiaries when they were not (Das et al., 2011). This implies that the treatment group included control women. Because of this and the possible selection bias, previous results are likely to have substantial downward biases.

We use the fourth round of DLHS (DLHS-4), which was surveyed in 2013-2014 when JSY was a mature program. It is unlikely that DLHS-4 has such extensive problems. We expect statistically significant effects on all intended outcomes. 
However, the objective of our paper is not only estimating JSY's effects on the intended outcomes but also to decompose those effects into the direct and indirect effects. There is no doubt that being a part of JSY's cash incentive an intended outcome can be affected by JSY directly. JSY can also affect it indirectly by changing another intended outcome if two outcomes are connected. We shall capture here both direct and indirect effects on the intended outcomes, and shall see which effects are stronger than the others. We do attempt these decompositions because policymakers will understand how JSY affects the intended outcomes, and then they will be able to take necessary actions to improve them further, and even they may save money to get the same anticipated effects. For example, if JSY works only to increase the utilization of an ANC service, and the utilization of institutional delivery service increases due to the increase in the utilization of an ANC service, JSY may not require to provide extra cash incentives to increase the utilization of institutional delivery service.

In this study, we consider two main intended outcomes - ANC and institutional delivery. We assume that both are connected, and in addition to the direct effect, JSY may have an indirect impact on each through each other. To capture both direct and indirect effects, we initially derive mathematical equations from raw differentials and then develop econometric models to estimate parameters in mathematical equations.

After this introductory section, we briefly discuss the program, JSY, in Section II. Section III formulates both theoretical and econometric models. Section IV describes data, and Section V presents results. Finally, Section VI contains the discussion and conclusion of the study.

\section{The Program}

India is steadily improving concerning reducing maternal and child mortality (Tandon, 2016; Registrar General of India, 2013). In spite of the steady progress, it still suffers from the highest underfive child mortality rate in the world (Tandon, 2016). The number of maternal deaths in India is higher than such amounts in its close competitors such as Indonesia and Malaysia (UNICEF, 2017). Moreover, the national figures hide the situation at the state level. In general, the northern states have very high maternal and child mortality rates in comparison to the southern states (Registrar General of India, 2013).

In the above setting, on 12th April 2005, the world's largest DSF program, JSY, was launched under the National Rural Health Mission (NRHM) by the prime minister of India. The primary objective of JSY is to increase the institutional delivery, but the ultimate goal of it is reducing maternal and neonatal mortality through increasing the institutional delivery. Along with the financial support of expanding the institutional delivery, JSY also gives financial incentives to increase the uptakes of at least three ANC services including a TT injection and IFA, and at least one PNC service for a mother and her child.

JSY is a universal program in 10 Low Performing States (LPS) - Uttar Pradesh, Uttaranchal, Bihar, Jharkhand, Madhya Pradesh, Chhattisgarh, Assam, Rajasthan, Orissa, and Jammu and Kashmir where the rates of institutional delivery are very low. In LPS, every pregnant woman who wants to deliver her child in a public or an accredited private hospital is eligible for JSY cash incentives. In other states, which are called High Performing States (HPS), the program gives financial incentives to only socio-economically disadvantaged women. Table (1) lists all eligibility criteria by state type. First, three criteria in HPS are the key criteria which determine whether a woman falls into the socio-economically disadvantaged group. A woman will be selected for JSY if she fulfills any one or all criteria in addition to fulfilling the other criteria. Criteria (4) and (6) in HPS are set to discourage baby boom, and (5) is a cost-effective criterion. It should be noted that the age criterion has been withdrawn recently. However, at the time of our data, the criterion remained and so was kept in this study.

\begin{tabular}{|l|l|}
\hline State Type & Eligibility Criteria \\
\hline LPS & $\begin{array}{l}\text { All pregnant women delivering in public or accredited } \\
\text { private institutions. }\end{array}$ \\
\hline HPS & $\begin{array}{l}\text { (1) Household of the pregnant woman has below poverty line } \\
\text { card, or/and }\end{array}$ \\
\hline & $\begin{array}{l}\text { (2) Household of the pregnant woman is scheduled caste, } \\
\text { or/and }\end{array}$ \\
\hline & (3) Household of the pregnant woman is tribe, and \\
\hline & (4) The pregnant woman aged 19 years and above, and \\
\hline & $\begin{array}{l}\text { (5) Give birth in public or accredited private institutions, and } \\
\text { second birth. }\end{array}$ \\
\hline Source: Ministry of Health and Family Welfare, India (Yojana, 2006). \\
\hline
\end{tabular}

\section{Table 1: Eligibility Criteria of JSY}

There are many field workers in the program who are appointed to work as intermediaries between the program administrators and the pregnant women. A field worker is called an accredited social health activist (ASHA). ASHAs identify the eligible pregnant women for JSY, and they help them from birth registration to the PNC service. They also prompt women to use the institutional delivery service. An ASHA receives cash incentives for his/her activities. Table 2 provides the information of cash incentives of JSY (in Indian Rupees) for both an eligible woman and an ASHA. LPS mothers receive a higher amount of cash than HPS mothers, in both rural and urban areas. ASHA's cash incentives do not vary by state type but vary by rural and urban areas. For both mothers and ASHAs, cash incentives are higher in the rural area than that in the urban area. This is probably the reason that the more destitute women live in the rural area, and rural transportation is costly.

\begin{tabular}{|l|l|l|l|l|l|l|}
\hline & \multicolumn{3}{|c|}{ Rural Area } & \multicolumn{3}{c|}{ Urban Area } \\
\hline State Type & Mother & ASHA & Total & Mother & ASHA & Total \\
\hline LPS & 1,400 & 600 & 2,000 & 1,000 & 400 & 1,400 \\
\hline HPS & 700 & 600 & 1,300 & 600 & 400 & 1,000 \\
\hline
\end{tabular}

Source: Ministry of Health and Family Welfare Annual Report 2016-2017, Government of India (MoHFW, 2017).

Table 2: Scale of Cash Assistance of JSY (in Indian Rupees (INR), and $64 \mathrm{INR}=1 \mathrm{USD})$.

An eligible pregnant woman aiming to deliver her baby in the public health institute receives her entire cash entitlement in one go at the health institution. An accredited private health institute reimburses the thirdfourth of the cash incentive in the first visit of an eligible woman who aims to deliver her baby there. A qualified woman also receives at least 250 rupees for transportation costs, which may vary by location and state. Up to an additional 1,500 rupees is given to her in the case of the caesarian section or obstetric complications. If she wants to deliver at home, she only receives 500 rupees. An ASHA's cash payment is made in two installments. The first part of the payment is given when he/she goes to the health institute with the pregnant women for the first time, and the second part of the payment is given after the PNC services taken by a mother.

\section{Methods}

\section{A. Mathematical Models}

Let $t$ and $d$ denote dummies for JSY and institutional delivery respectively. $t$ contains 1 if a mother is JSY recipient, or 0 if she is a nonrecipient of JSY and other similar cash incentives. $d$ has 1 if a mother gave her last birth in a health institute (e.g. hospital), or 0 if she gave her last birth at home. $A(t, d)$ denotes a dummy for antenatal care (ANC) given the status of $t$ and $d .1$ in $A(t, d)$ implies that at least one ANC is taken by a mother, and 0 implies that no ANC is taken by her. $N(t, d)$ is the number of mothers given the status of $t$ and $d$. We can write $N(t)=$ $N(t, d=1)+N(t, d=0)$ and $N(d)=N(t=1, d)+N(t=0, d)$. We can also write $A(t=1, d=1)=A(1,1), A(t=1, d=0)=A(1,0)$, $A(t=0, d=1)=A(0,1)$, and $A(t=0, d=0)=A(0,0)$. In a similar way, we can write the notations of the different combinations of $N(t, d)$. 
Case 1: The overall or total effect of JSY on ANC

Say, $\rho_{a}$ is the parameter of the effect of JSY on ANC, and we can write it, as $\rho_{a}=\overline{A(t=1)}-\overline{A(t=0)}$ as a raw differential. After some manipulation (see proof 1 in Appendix A), we can write it as

$$
\rho_{a}=\frac{\left(1+k_{0}\right) \overline{A(1,1)}-\left(1+k_{1}\right) \overline{A(0,1)}}{\left(1+k_{1}\right)\left(1+k_{0}\right)}+\frac{k_{1}\left(1+k_{0}\right) \overline{A(1,0)}-k_{0}\left(1+k_{1}\right) \overline{A(0,0)}}{\left(1+k_{1}\right)\left(1+k_{0}\right)},
$$

where $\rho_{a} \geq 0, \quad$ if $\quad\left(\left(1+k_{0}\right) \overline{A(1,1)}-\left(1+k_{1}\right) \overline{A(0,1)}\right) \geq 0$ and $\left(k_{1}\left(1+k_{0}\right) \overline{A(1,0)}-k_{0}\left(1+k_{1}\right) \overline{A(0,0)}\right) \geq 0 . \rho_{a} \geq 0$ even if one part is negative, but the positive part should be at least equal to the absolute value of the negative part. However, our main issue is that equation (1) captures the total effect of JSY on ANC, where JSY affects ANC in a direct way if $\overline{A(1,1)}>\overline{A(0,1)}$ and $\overline{A(1,0)}>$ $\overline{A(0,0)}$, and also in an indirect way via increasing the institutional delivery if $k_{0}>k_{1}$. One may argue that if JSY increases the institutional delivery, $k_{1}\left(1+k_{0}\right) \overline{A(1,0)}-k_{0}\left(1+k_{1}\right) \overline{A(0,0)}$ will decrease though $\left(1+k_{0}\right) \overline{A(1,1)}-\left(1+k_{1}\right) \overline{A(0,1)}$ will increase, but the total effect, $\rho_{a}$, will increase, as the rate of increasing part is stronger than the rate of decreasing part (see proof 2 in Appendix A). In the next two cases, we shall decompose the total effect into two effects.

Case 2: The effect of JSY on ANC, when JSY has no direct effect on ANC, but has a direct effect on the institutional delivery.

We can now show that $\rho_{a}>0$ even if $\overline{A(1,1)}=\overline{A(0,1)}$ and $\overline{A(1,0)}=$ $\overline{A(0,0)}$. It means that the effect of JSY on ANC can be positive even if there are no mean differences of the uptakes of ANC between JSY and non-JSY mothers in both institutional and home delivery cases. In other words, there is no direct effect of JSY on ANC in the case of both institutional delivery and home delivery. The effect of JSY can still be seen on ANC because of an increase in the institutional delivery, which is the main objective JSY. If the institutional delivery increases, $k_{0}>k_{1}$, which means that the ratio of the home delivery to the institutional delivery is higher in non-JSY mothers than that in JSY mothers, and in other words, the proportion of mothers with the institutional delivery is higher in JSY recipients than that in non-JSY recipients. We can now write (see proof 3, 4 and 5 in Appendix A),

$\rho_{a}=\delta_{a} \rho_{d}$,

where the indirect effect of JSY on ANC is the multiplication of the effect of JSY on the institutional delivery $\left(\rho_{d}\right)$ and the effect of institutional delivery on ANC $\left(\delta_{a}\right)$. That means that JSY indirectly affects ANC through increasing the institutional delivery. It should be noted that $\rho_{a}$ will be statistically significant, if $\delta_{a}$ or $\rho_{d}$ are statistically significant, as we can express the null hypothesis as, $H_{0}: \delta_{a} \rho_{d}=0$ or $\delta_{a}=0$ or $\rho_{d}=0$.

Case 3: The direct effect of JSY on ANC, when JSY has no effect on the institutional delivery.

Now, we assume that JSY cannot increase the institutional delivery, and therefore, $k_{0}-k_{1}=0$, or $k_{0}=k_{1}$. However, JSY may have a direct effect on ANC in the following way (see the proof 6 in Appendix A),

$\rho_{a}=w_{1} \rho_{a 1}+w_{0} \rho_{a 0}$,

where $\quad w_{1}=\frac{1}{\left(1+k_{0}\right)}=\frac{N(0,1)}{N(t=0)}, \quad w_{0}=\frac{k_{0}}{\left(1+k_{0}\right)}=\frac{N(0,0)}{N(t=0)}, \quad \rho_{a 1}=$ $(\overline{A(1,1)}-\overline{A(0,1)})$, and $\rho_{a 0}=(\overline{A(1,0)}-\overline{A(0,0)}) \cdot \rho_{a}$ is the weighted average of $\rho_{a 1}$, which is the parameter of the direct effect of JSY on ANC among mothers who delivered at the health institution, and $\rho_{a 0}$, which is the parameter of the direct effect of JSY on ANC among mothers who delivered at home. The weight, $w_{1}$, is the proportion of non-JSY mothers delivered at the health institution, and the weight, $w_{0}$, is the proportion of non-JSY mothers delivered at home. In our data, $w_{1}>w_{0}$, because most of the mothers delivered at health institutions. Here, $\rho_{a} \geq 0$, if $\rho_{a 1} \geq 0$ and $\rho_{a 0} \geq 0$. $\rho_{a}$ can be positive even if anyone of $\rho_{a 1}$ and $\rho_{a 0}$ is negative, but the positive part should outweigh the negative part.

\section{B. Econometric Models}

\section{Antenatal care}

We shall estimate $\rho_{a}$, the parameter of the effect of JSY on ANC, in three cases, as mentioned in the previous subsection. In the first case, we can estimate it directly from a regression. In the other two cases, we have to estimate it from other parameters estimated from regressions. In the second case, we shall estimate $\rho_{d}$ and $\delta_{a}$ from regressions, and their multiplication will give us the estimated value of $\rho_{a}$. We shall estimate $\rho_{a 1}$ and $\rho_{a 0}$ from regressions in the third case, and their weighted average will be the estimated value of $\rho_{a}$. We shall consider the following regression models in three cases.

In the following regressions, $A_{i}$ is a dummy of ANC of mother $i, d_{i}$ is a dummy of institutional delivery of mother $i, t_{i}$ is a JSY dummy of mother $i$, and $X_{i}$ is a vector of covariates (also called control variables, or explanatory variables, or predictors) of mother $i ; \alpha_{0}, \alpha_{1}, \alpha_{2}, \alpha_{3}$, and $\alpha_{4}$ are constant terms in relevant regressions; and $\rho_{a}, \rho_{d}, \delta_{a}, \rho_{a 1}$, and $\rho_{a 0}$ are relevant coefficients of the key variables; and $\hat{\beta}_{0}, \hat{\beta}_{1}, \hat{\beta}_{2}, \hat{\beta}_{3}$, and $\hat{\beta}_{4}$ are column vectors of parameters of $X_{i}$; and $\varepsilon_{0 i}, \varepsilon_{1 i}, \varepsilon_{2 i}, \varepsilon_{3 i}$, and $\varepsilon_{4 i}$ are error terms of mother $i$.

\section{Case 1:}

$$
A_{i}=\alpha_{0}+\rho_{a} t_{i}+X_{i} \dot{\beta}_{0}+\varepsilon_{0 i},
$$

The estimated value of $\rho_{a}$ in equation (4) will give us the total effect of JSY on ANC.

Case 2:

$$
\begin{aligned}
d_{i}= & \alpha_{1}+\rho_{d} t_{i}+X_{i} \dot{\beta}_{1}+\varepsilon_{1 i}, \\
& A_{i}=\alpha_{2}+\delta_{a} d_{i}+X_{i} \dot{\beta}_{2}+\varepsilon_{2 i} \text { if } t_{i}=0,
\end{aligned}
$$

where $\rho_{a}=\delta_{a} \rho_{d}$, and the estimated value of $\rho_{a}$ will give us the indirect effect of JSY on ANC.

Case 3:

$$
\begin{gathered}
A_{i}=\alpha_{3}+\rho_{a 1} t_{i}+X_{i} \dot{\beta}_{3}+\varepsilon_{3 i} \text { if } d_{i}=1, \\
A_{i}=\alpha_{4}+\rho_{a 0} t_{i}+X_{i} \hat{\beta}_{4}+\varepsilon_{4 i} \text { if } d_{i}=0,
\end{gathered}
$$

where $\rho_{a}=w_{1} \rho_{a 1}+w_{0} \rho_{a 0}$, the estimated value of $\rho_{a}$ will give us the direct effect of JSY on ANC.

\section{Institutional delivery}

We can do the similar analyses for the institutional delivery. In the case of antenatal care, we have analyzed that JSY can increase at least one ANC directly and also indirectly through increasing the institutional delivery as it has an effect on ANC. In that case, we have assumed that an increase in the demand for the final or intermediary product such as the institutional delivery can increase the demand for the primary product such as ANC. Now, we also assume that ANC and institutional delivery are mutually related, which means that an increase in demand for ANC can also increase the demand for the institutional delivery. In a similar way, we can also estimate the total effect, the indirect effect, and the direct effect of JSY on the institutional delivery. We run regressions in three cases as follows. Two regression equations will be same as in the previous case. Three additional equations will be here with additional constant terms $\left(\alpha_{5}\right.$, $\left.\alpha_{6}, \alpha_{7}\right)$, parameters $\left(\delta_{d}, \rho_{d 1}, \rho_{d 2}\right)$, and error terms $\left(\varepsilon_{5 i}, \varepsilon_{6 i}, \varepsilon_{7 i}\right)$.

\section{Case 1:}

$$
d_{i}=\alpha_{1}+\rho_{d} t_{i}+X_{i} \dot{\beta}_{1}+\varepsilon_{1 i}
$$

The estimated value of $\rho_{d}$ in equation (5) will give us the total effect of JSY on the institutional delivery.

Case2:

$$
\begin{aligned}
& A_{i}=\alpha_{0}+\rho_{a} t_{i}+X_{i} \dot{\beta}_{0}+\varepsilon_{0 i}, \\
& d_{i}=\alpha_{5}+\delta_{d} A_{i}+X_{i} \dot{\beta}_{5}+\varepsilon_{5 i} \text { if } t_{i}=0,
\end{aligned}
$$

where $\rho_{d}=\delta_{d} \rho_{a}$, and the estimated value of $\rho_{d}$ will give us indirect effect of JSY on institutional delivery.

Case 3:

$$
\begin{aligned}
& d_{i}=\alpha_{6}+\rho_{d 1} t_{i}+X_{i} \dot{\beta}_{6}+\varepsilon_{6 i} \quad \text { if } A_{i}=1, \\
& d_{i}=\alpha_{7}+\rho_{d 0} t_{i}+X_{i} \dot{\beta}_{7}+\varepsilon_{7 i} \quad \text { if } A=0,
\end{aligned}
$$

where $\rho_{d}=k_{1} \rho_{d 1}+k_{0} \rho_{d 0}$, the estimated value of $\rho_{d}$ will give us the direct effect of JSY on the institutional delivery. Here, the weight, $k_{1}$, is the proportion of non-JSY mothers having at least one ANC, and the weight, $k_{0}$, is the proportion of non-JSY mothers having no ANC. 
Data

Data from the fourth round of the District Level Household Survey (DLHS-4) was used, which was surveyed in 2013-14. Because of surveying different households in different waves, DLHS is considered as a repeated cross-section. We do not use other previous waves of it. DLHS-1 and DLHS-2 were surveyed before the implementation of JSY, and DLHS-3 was surveyed immediately after the implementation of JSY. DLHS-3 misclassified many non-JSY beneficiaries as JSY beneficiaries (Das et al., 2011) and therefore, it is likely to produce biased results.

DLHS-4 collected data from 378,487 households located in 18 highperforming states and 3 high performing territories. However, for the data on the maternal and child health care, the latest wave surveyed only 86,000 women who gave their last births from 2008. The information about maternal and child health care was collected from only their last births. So, the times of all last births surveyed by DLHS-4 fell within the period of JSY's proper implementation (2007present). Therefore, as opposed to DLHS-3, DLHS-4 has a less chance of misclassifying untreated women as treated.

Among 86,000 women, about $22 \%$ responded as JSY beneficiaries, and about $4 \%$ were the beneficiaries of other schemes.
We dropped other schemes' recipients from our data, as the study's focus is JSY's evaluation. Besides, they may make our results biased, as those schemes might have different cash incentives. In this way, a significant amount of data has been dropped. Moreover, many women have been dropped automatically from the data, as they did not respond to the questions on variables used in this study (e.g., missing value problem).

In Table 3, we present means of both outcome variables (A and d) and covariates by the JSY treatment status (t). It should be noted that covariates include both the program eligibility criteria such as poverty dummy, scheduled caste dummy, and tribe dummy and the self-selection criteria such as wealth index, age, education, religion, and residence. Among these covariates, education and age are individual characteristics, and all others are women's household characteristics. Other than wealth index, all variables are generated in straight away from the data. Using the principal component analysis, wealth index is generated from the availability of a household's assets, which include cooking fuel, house type, number of dwelling rooms, electricity, house ownership, landholding, radio, television, computer, internet, telephone, mobile phone, washing machine, refrigerator, sewing machine, watch, bicycle, motorcycle, car, tractor, tube well, cart and air cooler.

\begin{tabular}{|c|c|c|c|c|c|c|}
\hline & \multicolumn{2}{|c|}{ JSY $(t=1)$} & \multicolumn{2}{|c|}{ Non-JSY $(t=0)$} & \multirow[b]{2}{*}{ Difference } & \multirow[b]{2}{*}{ p value } \\
\hline & Mean & $\boldsymbol{N}$ & Mean & $\boldsymbol{N}$ & & \\
\hline$t$ & 1 & 15,849 & 0 & 57,265 & & \\
\hline$A$ & 0.949 & 15,849 & 0.826 & 57,263 & 0.122 & 0.000 \\
\hline$d$ & 0.935 & 15,848 & 0.773 & 57,262 & 0.162 & 0.000 \\
\hline \multicolumn{7}{|l|}{ Socio-economic conditions (covariates into $X$ ) } \\
\hline Wealth index & -0.655 & 15,843 & -0.016 & 57,230 & -0.639 & 0.000 \\
\hline Age of mother (in year) & 26.106 & 15,849 & 27.440 & 57,265 & -1.334 & 0.000 \\
\hline Years of education completed by mother & 8.674 & 13,669 & 9.563 & 47,641 & -0.889 & 0.000 \\
\hline Years of education completed by husband & 8.812 & 14,036 & 9.821 & 50,457 & -1.009 & 0.000 \\
\hline Hindu (yes $=1$, otherwise $=0$ ) & 0.698 & 15,847 & 0.653 & 57,249 & 0.045 & 0.000 \\
\hline Scheduled caste $($ yes $=1$, otherwise $=0$ ) & 0.310 & 15,149 & 0.221 & 53,951 & 0.089 & 0.000 \\
\hline Tribe $($ yes $=1$, otherwise $=0$ ) & 0.177 & 15,841 & 0.176 & 57,185 & 0.001 & 0.587 \\
\hline Poor $($ yes $=1$, otherwise $=0$ ) & 0.469 & 15,846 & 0.318 & 57,246 & 0.151 & 0.000 \\
\hline Birth order & 1.842 & 15,793 & 2.162 & 56,822 & -0.320 & 0.000 \\
\hline Rural (yes $=1$, otherwise $=0$ ) & 0.683 & 15,849 & 0.593 & 57,265 & 0.090 & 0.000 \\
\hline
\end{tabular}

\section{Table 3: Summary statistics.}

However, Table 3 also presents the number of observations with each mean. Differences of means between JSY and non-JSY women are also shown with their $\mathrm{p}$ values. Combining JSY and non-JSY women, there are around 73,000 women in most of the cases. Among them, around $22 \%$ are treated by JSY. We see that almost all variables have significantly different means between JSY and non-JSY women, as $\mathrm{p}$ values are approximately zero. We expect such results in the case of $A$ and $d$, but not in covariates. Otherwise, our results are supposed to be biased due to the non-randomization. As almost all covariates are statistically and significantly different between JSY and non-JSY women, the non-randomization exists in our data. However, we expect that the propensity score matching (PSM) will remove the nonrandomization.

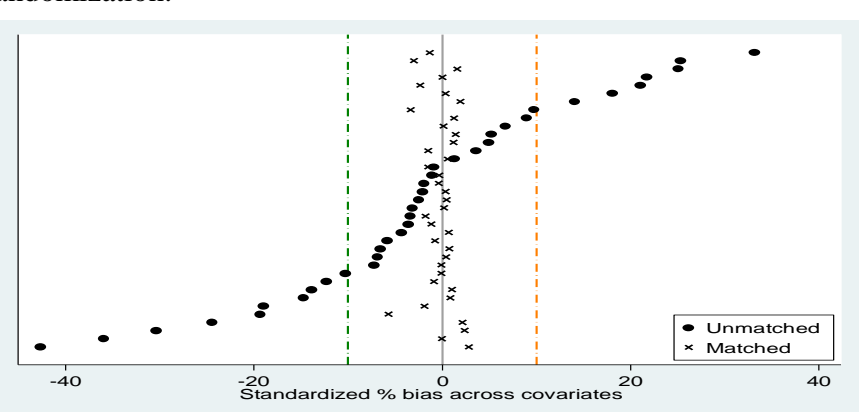

Figure 1: Biases of covariates between JSY and non-JSY women.
Note: Data are from DLHS-4. Biases of covariates give a better indication of the randomization than means differences of covariates. In a bias calculation, a mean difference of a covariate between the treatment and control group is standardized by the standard errors. Bias calculation is slightly different for a continuous covariate and a dummy covariate (Thomas, 2003). Say, $x$ is a continuous covariate, $\bar{x}_{1}$ is the mean of it in the treatment group (JSY recipients), $\bar{x}_{0}$ is the mean of it in the control group (non-JSY recipients), $s_{1}^{2}$ is the variance of it in the treatment group (JSY recipients), and $s_{0}^{2}$ is the variance of it in the control group (non-JSY recipients). So, Bias $_{x}=\frac{\left(\bar{x}_{1}-\bar{x}_{0}\right)}{\sqrt{\left(s_{1}^{2}+s_{0}^{2}\right) / 2}}$. Say, $d$ is the dummy variable, $\hat{p}_{1}$ is the mean of it in the treatment group (JSY recipients), which can also be said as the probability of $d=1$ in the treatment group. Similarly, $\hat{p}_{0}$ is the probability of $d=1$ in the control group. So, Bias $_{d}=\frac{\left(\hat{p}_{1}-\hat{p}_{0}\right)}{\sqrt{\left\{\hat{p}_{1}\left(1-\hat{p}_{1}\right)+\hat{p}_{0}\left(1-\hat{p}_{0}\right)\right\}} / 2}$. In the figure, biases are estimated before and after running the propensity score matching. Dots are biases without matching, and crosses are biases with matching. There is a criterion that if biases remain between -10 and 10 , the randomization exists in data (Thomas, 2003). In the figure, the green reference line is at -10 and the orange reference line is at 10 . All biases with matching fall between these two lines, which indicate the randomization with matching. So, our PSM produces unbiased results in the case of randomization issue.

In Figure 1, we show biases of covariates with PSM and without PSM. Biases are standardized means' differences between the treatment and the control groups and are better indicators of the randomization. 
If biases are within the range of -10 and 10, the randomization exists (Thomas, 2003). We see in the figure that after matching, all biases fall within this range. Thus, our PSM produces unbiased results converting the data into the randomization.

\section{Results}

For three different cases, we estimate the effects of JSY on ANC and the institutional delivery. As we know, the first case shows the overall effects, the second case shows the indirect effects, and the third case shows the direct effects.
For comparisons, in every case, we run linear probability models (OLS regressions) and logit regressions in addition to the PSM regressions and raw estimates. To maintain the overlapping assumption, we also run PSM regressions for subsamples identified when the propensity score is between 0.10 and 0.90 (Crump et al.; 2009). Thus, PSM with overlapping produces the most reliable estimates here. In the case of PSM, we use the default options in Stata's psmatch2 command meaning that we run the nearest neighbor PSM.

\begin{tabular}{|c|c|c|c|c|c|c|c|}
\hline & \multicolumn{7}{|c|}{ Antenatal Care } \\
\hline & \multirow{2}{*}{$\frac{\widehat{\rho}_{a}}{\text { Case } 1}$} & \multicolumn{3}{|c|}{ Case 2} & \multicolumn{3}{|c|}{ Case 3} \\
\hline & & (2) $\widehat{\rho_{d}}$ & (3) $\widehat{\delta_{a}}$ & (4) ${ }^{\widehat{\rho_{a}}}$ & (5) & (6) $\widehat{\widehat{\rho_{a 0}}}$ & (7) ${ } \widehat{\rho_{a}}$ \\
\hline Raw & $\begin{array}{l}0.122^{* * *} \\
(0.002)\end{array}$ & $\begin{array}{l}0.162^{\text {*** }} \\
(0.003)\end{array}$ & $\begin{array}{l}0.377^{* * * *} \\
(0.005)\end{array}$ & $0.061^{\text {**** }}$ & $\begin{array}{l}0.042^{* * * *} \\
(0.002)\end{array}$ & $\begin{array}{l}0.336^{* * * *} \\
(0.011)\end{array}$ & $0.109^{* * * *}$ \\
\hline OLS & $\begin{array}{l}0.079^{\text {***** }} \\
(0.003)\end{array}$ & $\begin{array}{l}0.124^{\text {***** }} \\
(0.003)\end{array}$ & $\begin{array}{l}0.223^{* * * * *} \\
(0.006)\end{array}$ & $0.028^{* * *}$ & $\begin{array}{l}0.037^{\text {****** }} \\
(0.003)\end{array}$ & $\begin{array}{l}0.167^{\text {***** }} \\
(0.015)\end{array}$ & $0.067^{* * *}$ \\
\hline Logit & $\begin{array}{l}0.052^{\text {**** }} \\
(0.002)\end{array}$ & $\begin{array}{l}0.059^{* * * *} \\
(0.002)\end{array}$ & $\begin{array}{l}0.145^{* * *} \\
(0.000)\end{array}$ & $0.009^{* * *}$ & $\begin{array}{l}0.026^{* * * *} \\
(0.002)\end{array}$ & $\begin{array}{l}0.221^{\text {*a* }} \\
(0.018)\end{array}$ & $0.070^{* * * *}$ \\
\hline Matching & $\begin{array}{l}0.079^{\text {**** }} \\
(0.004)\end{array}$ & $\begin{array}{l}0.127^{\text {**** }} \\
(0.005)\end{array}$ & $\begin{array}{l}0.177^{\text {*a* }} \\
(0.015)\end{array}$ & $0.021^{\text {***** }}$ & $\begin{array}{l}0.041^{\text {* }} \text { (0.004) } \\
(0.004)\end{array}$ & $\begin{array}{l}0.135^{\text {**** }} \\
(0.022)\end{array}$ & $0.069^{* * * *}$ \\
\hline \multirow[t]{4}{*}{ Matching with overlapping } & $\begin{array}{l}0.074^{\text {**** }} \\
(0.005)\end{array}$ & $\begin{array}{l}0.123^{* * * *} \\
(0.005)\end{array}$ & $\begin{array}{l}0.195^{\text {**** }} \\
(0.012)\end{array}$ & $0.024^{\text {***** }}$ & $\begin{array}{l}0.042^{\text {**** }} \\
(0.004)\end{array}$ & $\begin{array}{l}0.077^{\text {**** }} \\
(0.027)\end{array}$ & $0.050^{* * * *}$ \\
\hline & \multicolumn{7}{|c|}{ Institutional Delivery } \\
\hline & Case 1 & \multicolumn{3}{|l|}{ Case 2} & \multicolumn{3}{|l|}{ Case 3} \\
\hline & (1) $\widehat{\widehat{\rho_{d}}}$ & (2) $\widehat{\rho_{a}}$ & (3) $\widehat{\widehat{\delta_{d}}}$ & (4) $\widehat{\widehat{\rho_{d}}}$ & (5) ${ }^{\widehat{\rho_{d 1} 1}}$ & (6) ${ }^{\widehat{\rho_{d 0}}}$ & (7) $\widehat{\widehat{\rho_{d}}}$ \\
\hline Raw & $\begin{array}{l}0.162^{* * * *} \\
(0.003)\end{array}$ & $\begin{array}{l}0.122^{\text {***** }} \\
(0.002)\end{array}$ & $\begin{array}{l}0.461^{* * * *} \\
(0.005)\end{array}$ & $0.056^{\text {***** }}$ & $\begin{array}{l}0.087^{* * * *} \\
(0.003)\end{array}$ & $\begin{array}{l}0.443^{* * * *} \\
(0.014)\end{array}$ & $0.149^{* * * *}$ \\
\hline OLS & $\begin{array}{l}0.124^{\text {***** }} \\
(0.003)\end{array}$ & $\begin{array}{l}0.079^{* * * *} \\
(0.003)\end{array}$ & $\begin{array}{l}0.233^{\text {**** }} \\
(0.006)\end{array}$ & $0.018^{* * * *}$ & $\begin{array}{l}0.091^{\text {***** }} \\
(0.003)\end{array}$ & $\begin{array}{l}0.261^{\text {K*** }} \\
(0.017)\end{array}$ & $0.121^{* * * *}$ \\
\hline Logit & $\begin{array}{l}0.059^{* * * *} \\
(0.002)\end{array}$ & $\begin{array}{l}0.052^{* * * *} \\
(0.002)\end{array}$ & $\begin{array}{l}0.146^{* * * *} \\
(0.007)\end{array}$ & $0.008^{* * * *}$ & $\begin{array}{l}0.046^{* * * *} \\
(0.002)\end{array}$ & $\begin{array}{l}0.375^{* * * *} \\
(0.019)\end{array}$ & $0.103^{* * *}$ \\
\hline Matching & $\begin{array}{l}0.127^{* * * *} \\
(0.005)\end{array}$ & $\begin{array}{l}0.079^{\text {**** }} \\
(0.004)\end{array}$ & $\begin{array}{l}0.143^{\text {*a* }} \\
(0.011)\end{array}$ & $0.012^{* * * *}$ & $\begin{array}{l}0.098^{* * * *} \\
(0.005)\end{array}$ & $\begin{array}{l}0.258^{* * * *} \\
(0.029)\end{array}$ & $0.126^{* * * *}$ \\
\hline Matching with overlapping & $\begin{array}{l}0.123^{\text {**** }} \\
(0.005)\end{array}$ & $\begin{array}{l}0.074^{\text {N*** }} \\
(0.005)\end{array}$ & $\begin{array}{l}0.263^{\text {*** }} \\
(0.019)\end{array}$ & $0.019^{* * * *}$ & $\begin{array}{l}0.104^{\text {**** }} \\
(0.005)\end{array}$ & $\begin{array}{l}0.214^{\text {**** }} \\
(0.034)\end{array}$ & $0.123^{* * * *}$ \\
\hline
\end{tabular}

Note: In all regressions, state dummies and (last) birth year dummies are also included as covariates. Standard errors are in parentheses. ${ }^{*},{ }^{* *}$, and imply that estimates are statistically significant at $10 \%, 5 \%$, and $1 \%$ significance levels respectively.

Table 4: Main results of antenatal care and institutional delivery in three cases.

In Table 4, we show the values of the key parameters , which are estimated using equations (2-11). In the first panel (under ANC), ( $\rho_{-} a$ )$^{\wedge}$ in case $1,\left(\rho \_d\right)^{\wedge}$ and $\left(\delta \_a\right)^{\wedge}$ in case 2 , and $\left(\rho \_a 1\right)^{\wedge}$ and $\left(\rho \_a 0\right)^{\wedge}$ in case 3 are estimated from regressions of equations (4-8), which are run using the regression methods mentioned above. Similarly, in the second panel (under institutional delivery $),\left(\rho \_d\right)^{\wedge}$ in case $1,\left(\rho \_a\right)^{\wedge}$ and $\left(\delta \_d\right)^{\wedge}$ in case 2 , and $\left(\rho \_d 1\right)^{\wedge}$ and $\left(\rho \_d 0\right)^{\wedge}$ in case 3 are estimated from regressions of equations $(4,5,9-11)$ which are run using those regression methods. All regressions have used covariates listed in Table 3 plus state dummies and birth year dummies. Standard errors of those parameters estimated from regressions are reported in parentheses. All other parameters are estimated without regressions but following equations (2) and (3), and therefore, they do not have any standard errors . For example, $\left(\rho \_a\right)^{\wedge}$ in case 2 under ANC is estimated using equation (2). This is a measure of indirect effects of JSY on ANC. ( $\left.\rho \_a\right)^{\wedge}$ in case 3 under ANC is estimated using equation (3). This is a measure of the direct effects of JSY on ANC . Similarly, $\left(\rho \_d\right)^{\wedge}$ in case 2 under institutional delivery is estimated following equation (2) (meaning similar to equation (2)). This is a measure of indirect effects of JSY on the institutional delivery . $\left(\rho \_d\right)^{\wedge}$ in case 3 under institutional delivery is estimated following equation (3) (that means as the similar way of equation (3)). This is a measure of the direct effects of JSY on the institutional delivery. Each regression type shows that the direct effects of JSY are stronger than the indirect effects, in both ANC and institutional delivery.

\section{Discussion and Conclusion}

JSY provides cash incentives to eligible pregnant women to increase both ANC and the institutional delivery. Both of these maternal outcomes are under the continuum of maternal care, and therefore, they are connected with each other. JSY effects each indirectly through the other. If their connections become stronger, the indirect effects will be stronger. Indirect effects also depend on the direct effects of JSY. If we see the numbers in the case of PSM with overlapping in column (3) of Table 4, we understand that the effect of ANC on the institutional delivery is stronger than the reverse effect. The direct effect of JSY on the institutional delivery is much stronger than the direct effect of JSY on ANC (see column (7) of Table (4)). Therefore, the overall effect of JSY on the institutional delivery is much stronger than the overall effect of JSY on ANC (see columns (1) and (2) in Table (4)). Correspondingly, the indirect effect of JSY on the institutional delivery is weaker than the indirect effect of JSY on ANC (see column (4) of Table (4)), in spite of a higher effect of ANC on the institutional delivery than the reverse effect.

From a policy perspective, we can say that policymakers should work first to increase the uptake of ANC, and then the institutional delivery will automatically increase as a result of their connections. In Table (3), we can see that the uptake of ANC is 0.949 or $94.9 \%$ amongst the JSY women. In other words, we can say that $5.1 \%$ of JSY women do not take any ANC service. Policymakers should identify why some of JSY women do not take any ANC service, in spite of cash incentives they receive. 
If cash incentives are insufficient for them, that should be increased so that the uptake of ANC becomes $100 \%$. When women go to health institutions for any ANC service, health professionals should strongly recommend them to have the institutional delivery. In this way, the effect of ANC on the institutional delivery will increase, and thus, the indirect effect and then the overall effect of JSY on the institutional delivery will increase. Policymakers should also identify why $6.5 \%$ of JSY women do not go to health institutions for the institutional delivery (see Table (3)). The uptake of the institutional delivery among JSY women should be $100 \%$ too, as it is the JSY's main objective.

In this paper, we decompose the effects of JSY on ANC and the institutional delivery. In addition to the methodological contribution, we consider this as an important contribution. After this decomposition, policymakers will now understand how JSY affects ANC and the institutional delivery and how much efforts should be taken to improve them.

Compliance with Ethical Standards

Funding: This study was funded by Medical Research Council (MRC), UK (grant number MR/N006267/1).

Conflict of Interest: The authors declare that they have no conflict of interest.

\section{Appendix A}

$$
\begin{aligned}
& \text { Proof1: } \rho_{a}=\overline{A(t=1)}-\overline{A(t=0)} \\
& =\frac{\sum A(t=1)}{N(t=1)}-\frac{\sum A(t=0)}{N(t=0)}=\frac{\sum(A(t=1, d=1)+A(t=1, d=0))}{N(t=1, d=1)+N(t=1, d=0)} \\
& =\frac{\sum A(1,1)}{N(1,1)+N(1,0)}+\frac{\sum A(1,0)}{N(1,1)+N(1,0)}-\frac{\sum A(0,1)}{N(0,1)+N(0,0)}- \\
& \sum A(t=0, d=0) \\
& N(0,1)+N(0,0) \\
& =\frac{\sum A(1,1) / N(1,1)}{1+N(1,0) / N(1,1)}+\frac{\sum A(1,0) / N(1,0)}{N(1,1) / N(1,0)+1}- \\
& \frac{\sum A(0,1) / N(0,1)}{1+N(0,0) / N(0,1)}-\frac{\sum A(t=0, d=0) / N(0,0)}{N(0,1) / N(0,0)+1} \\
& \text { Let, } k_{1}=N(1,0) / N(1,1), k_{0}=N(0,0) / N(0,1) \text {. We then can write, } \\
& \rho_{a}=\frac{\overline{A(1,1)}}{1+k_{1}}+\frac{\overline{A(1,0)}}{1 / k_{1}+1}-\frac{\overline{A(0,1)}}{1+k_{0}}-\frac{\overline{A(0,0)}}{1 / k_{0}+1} \\
& = \\
& \frac{\left(1+k_{0}\right) \overline{A(1,1)}-\left(1+k_{1}\right) \overline{A(0,1)}}{\left(1+k_{1}\right)\left(1+k_{0}\right)}+\frac{\left(1+1 / k_{0}\right) \overline{A(1,0)}-\left(1+1 / k_{1}\right) \overline{A(0,0)}}{\left(1+1 / k_{1}\right)\left(1+1 / k_{0}\right)} \\
& = \\
& \frac{\left(1+k_{0}\right) \overline{A(1,1)}-\left(1+k_{1}\right) \overline{A(0,1)}}{\left(1+k_{1}\right)\left(1+k_{0}\right)}+ \\
& \frac{k_{1}\left(1+k_{0}\right) \overline{A(1,0)}-k_{0}\left(1+k_{1}\right) \overline{A(0,0)}}{\left(1+k_{1}\right)\left(1+k_{0}\right)} \text {. } \\
& \left(1+k_{1}\right)\left(1+k_{0}\right)
\end{aligned}
$$

Proof 2: We can proof it with differential calculus. If institutional delivery increases, either $k_{0}$ increases or $k_{1}$ decreases so that $k_{0}-k_{1}$ increases. Now, say $k_{0}$ increases and $k_{1}$ remains constant, then the increasing rate of $\left(1+k_{0}\right) \overline{A(1,1)}-\left(1+k_{1}\right) \overline{A(0,1)}$ will be $\overline{A(1,1)}$ (we get it if we differentiate $\left(1+k_{0}\right) \overline{A(1,1)}-\left(1+k_{1}\right) \overline{A(0,1)}$ by $\left.k_{0}\right)$, and the increasing rate of $k_{1}\left(1+k_{0}\right) \overline{A(1,0)}-k_{0}(1+$ $\left.k_{1}\right) \overline{A(0,0)}$ will be $k_{1} \overline{A(1,0)}-\left(1+k_{1}\right) \overline{A(0,0)}$ (we get it if we differentiate $k_{1}\left(1+k_{0}\right) \overline{A(1,0)}-k_{0}\left(1+k_{1}\right) \overline{A(0,0)}$ by $\left.k_{0}\right)$. It is likely that $\overline{A(1,1)}>k_{1} \overline{A(1,0)}-\left(1+k_{1}\right) \overline{A(0,0)}$, and thus, we can say that the total effect, $\rho_{a}$, will increase if institutional delivery increases.

$$
\begin{aligned}
& \text { Proof 3: } \rho_{a}=\frac{\left(1+k_{0}\right) \overline{A(1,1)}-\left(1+k_{1}\right) \overline{A(0,1)}}{\left(1+k_{1}\right)\left(1+k_{0}\right)}+\frac{k_{1}\left(1+k_{0}\right) \overline{A(1,0)}-k_{0}\left(1+k_{1}\right) \overline{A(0,0)}}{\left(1+k_{1}\right)\left(1+k_{0}\right)} \\
& =\frac{\left(1+k_{0}\right) \overline{A(0,1)}-\left(1+k_{1}\right) \overline{A(0,1)}}{\left(1+k_{1}\right)\left(1+k_{0}\right)}+\frac{k_{1}\left(1+k_{0}\right) \overline{A(0,0)}-k_{0}\left(1+k_{1}\right) \overline{A(0,0)}}{\left(1+k_{1}\right)\left(1+k_{0}\right)} \\
& =\frac{\left(k_{0}-k_{1}\right) \overline{A(0,1)}}{\left(1+k_{1}\right)\left(1+k_{0}\right)}+\frac{\left(k_{1}-k_{0}\right) \overline{A(0,0)}}{\left(1+k_{1}\right)\left(1+k_{0}\right)}
\end{aligned}
$$

$$
=(\overline{A(0,1)}-\overline{A(0,0)}) \frac{\left(k_{0}-k_{1}\right)}{\left(1+k_{1}\right)\left(1+k_{0}\right)} .
$$

In the above case, we know that $\left(k_{0}-k_{1}\right)>0$. Now, $\rho_{a} \geq 0$, if $(\overline{A(0,1)}-\overline{A(0,0)}) \geq 0$, which implies that in the case of non-JSY mothers, the uptakes of ANC must be higher in mothers with institutional delivery than that in mothers who deliver at home. We know that $\left(k_{0}-\right.$ $\left.k_{1}\right)>0$ if JSY program has a positive effect on institutional delivery, which means that mean difference of institutional delivery between JSY and non-JSY mothers is positive, e.g. $\overline{d(t=1)}-\overline{d(t=0)}>0$. We can show $\left(k_{0}-k_{1}\right)$ as a function of $\overline{d(t=1)}-\overline{d(t=0)}$, and that is $\left(k_{0}-k_{1}\right)=\frac{N(t=1) N(t=0)}{N(1,1) N(0,1)}(\overline{d(t=1)}-\overline{d(t=0)})=\frac{N(t=1) N(t=0)}{N(1,1) N(0,1)} \rho_{d}$

where $\rho_{d}=\overline{d(t=1)}-\overline{d(t=0)}$ (see proof 4). If we go further, we can write, $\rho_{a}=(\overline{A(1,1)}-\overline{A(1,0)}) \rho_{d}$ (see proof 5). By denoting $(\overline{A(1,1)}-$ $\overline{A(1,0)})=\delta_{a}$ that means that the parameter of the effect of institutional delivery on ANC, we can write, $\rho_{a}=\delta_{a} \rho_{d}$.

Proof 4: We have denoted $\rho_{d}$ as the effect of JSY on institutional delivery. For the time being, if we estimate it by using mean difference or raw differential, we can write it as follows,

$$
\begin{aligned}
\rho_{d} & =\overline{d(t=1)}-\overline{d(t=0)} \\
& =\frac{\sum d(t=1)}{N(1,1)+N(1,0)}-\frac{\sum d(t=0)}{N(0,1)+N(0,0)} \\
& =\frac{N(1,1)}{N(1,1)+N(1,0)}-\frac{N(0,1)}{N(0,1)+N(0,0)} \\
& =\frac{N(1,1)(N(0,1)+N(0,0))-N(0,1)(N(1,1)+N(1,0))}{(N(1,1)+N(1,0))(N(0,1)+N(0,0))} \\
& =\frac{N(1,1) N(0,1)+N(1,1) N(0,0)-N(0,1) N(1,1)-N(0,1) N(1,0)}{N(t=1) N(t=0)} \\
& =\frac{N(1,1) N(0,0)-N(0,1) N(1,0)}{N(t=1) N(t=0)} \\
& =\frac{N(1,1) N(0,0)-N(0,1) N(1,0)}{N(1,1) N(0,1)} \frac{N(1,1) N(0,1)}{N(t=1) N(t=0)} \\
& =\left(\frac{N(1,1) N(0,0)}{N(1,1) N(0,1)}-\frac{N(0,1) N(1,0)}{N(1,1) N(0,1)}\right) \frac{N(1,1) N(0,1)}{N(t=1) N(t=0)} \\
& =\left(\frac{N(0,0)}{N(0,1)}-\frac{N(1,0)}{N(1,1)}\right) \frac{N(1,1) N(0,1)}{N(t=1) N(t=0)} \\
& =\left(k_{0}-k_{1}\right) \frac{N(1,1) N(0,1)}{N(t=1) N(t=0)} \\
& =\left(k_{0}-k_{1}\right) \frac{N(1,1) N(0,1)}{N(t=1) N(t=0)} .
\end{aligned}
$$

From the above equation, we can write, $\left(k_{0}-k_{1}\right)=\frac{N(t=1) N(t=0)}{N(1,1) N(0,1)} \rho_{d}$.

Proof 5: If we substitute the value of $\left(k_{0}-k_{1}\right)$ from proof 4 into $\rho_{a}=(\overline{A(0,1)}-\overline{A(0,0)}) \frac{\left(k_{0}-k_{1}\right)}{\left(1+k_{1}\right)\left(1+k_{0}\right)}$ in proof 3, we have the following expression.

$$
\begin{gathered}
\rho_{a}=(\overline{A(1,1)}-\overline{A(1,0)}) \frac{1}{\left(1+k_{1}\right)\left(1+k_{0}\right)} \frac{N(t=1) N(t=0)}{N(1,1) N(0,1)} \rho_{d} \\
=(\overline{A(1,1)}-\overline{A(1,0)}) \frac{1}{(1+N(1,0) / N(1,1))(1+N(0,0) / N(0,1))} \frac{N(t=1) N(t=0)}{N(1,1) N(0,1)} \rho_{d} \\
=(\overline{A(1,1)}-\overline{A(1,0)}) \frac{1}{\left(\frac{N(1,1)+N(1,0)}{N(1,1)}\right)\left(\frac{N(0,1)+N(0,0)}{N(0,1)}\right)} \frac{N(t=1) N(t=0)}{N(1,1) N(0,1)} \rho_{d} \\
=(\overline{A(1,1)}-\overline{A(1,0)}) \frac{1}{\left(\frac{N(t=1)}{N(1,1)}\right)\left(\frac{N(t=0)}{N(0,1)}\right)} \frac{N(t=1) N(t=0)}{N(1,1) N(0,1)} \rho_{d} \\
=(\overline{A(1,1)}-\overline{A(1,0)}) \frac{1}{\frac{N(t=1) N(t=0)}{N(1,1) N(0,1)}} \frac{N(t=1) N(t=0)}{N(1,1) N(0,1)} \rho_{d} \\
=\left(\overline{A(1,1)}-\overline{A(1,0))} \frac{N(1,1) N(0,1)}{N(t=1) N(t=0)} \frac{N(t=1) N(t=0)}{N(1,1) N(0,1)} \rho_{d}\right. \\
=\left(\overline{A(1,1)}-\overline{A(1,0))} \rho_{d .}\right.
\end{gathered}
$$

Proof 6:

$$
\begin{aligned}
& \rho_{a}=\frac{\left(1+k_{0}\right) \overline{A(1,1)}-\left(1+k_{0}\right) \overline{A(0,1)}}{\left(1+k_{0}\right)\left(1+k_{0}\right)} \\
& +\frac{k_{0}\left(1+k_{0}\right) \overline{A(1,0)}-k_{0}\left(1+k_{0}\right) \overline{A(0,0)}}{\left(1+k_{0}\right)\left(1+k_{0}\right)} \\
& =\frac{\left(1+k_{0}\right)(\overline{A(1,1)}-\overline{A(0,1)})}{\left(1+k_{0}\right)\left(1+k_{0}\right)}+\frac{k_{0}\left(1+k_{0}\right)(\overline{A(1,0)}-\overline{A(0,0)})}{\left(1+k_{0}\right)\left(1+k_{0}\right)}
\end{aligned}
$$




$$
\begin{aligned}
& =\frac{(\overline{A(1,1)}-\overline{A(0,1)})}{\left(1+k_{0}\right)}+\frac{k_{0}(\overline{A(1,0)}-\overline{A(0,0)})}{\left(1+k_{0}\right)} \\
& =w_{1} \rho_{a 1}+w_{0} \rho_{a 0} .
\end{aligned}
$$

\section{References}

1. Anwar I, Sami, M, Akhtar, N, Chowdhury, M. Salma, U. (2008). Inequity in maternal health-care services: evidence from home-based skilled birth-attendant programmes in Bangladesh,

2. Behrman, J. R. and Knowles, J. C. (1998). Population and reproductive health: An economic framework for policy evaluation, pp. 697-737.

3. Bhatia, M. and Gorter, A. (2007). Improving access to reproductive and child health services in developing countries: are competitive voucher schemes an option?,19(7): 975-981.

4. Bhatia, M., Yesudian, C., Gorter, A. and Thankappan, K. (2006). Demand side financing for reproductive and child health services in india, pp. 279-284.

5. Carvalho, N., Thacker, N., Gupta, S. S. and Salomon, J. A. (2014). More evidence on the impact of India's conditional cash transfer program, janani suraksha yojana: quasiexperimental evaluation of the effects on childhood immunization and other reproductive and child health outcomes, 9(10): e109311.

6. Crump RK., Hotz VJ., Imbens GW. and Mitnik OA. (2009). Dealing with limited overlap in estimation of average treatment effects.pp. 1-13.

7. Engineer, C., Dale, E., Agarwal, A., Agarwal, A., Alonge, O., Edward, A., Gupta, S., Schuh, H., Burnham, G. and Peters, D. (2016). Effectiveness of a pay-for-performance intervention to improve maternal and child health services in Afghanistan: A cluster randomized trial, 45(2): 451-459.

8. Ensor, T., Chhun, C., Kimsun, T., McPake, B. and Edoka, I. (2017). Impact of health financing policies in cambodia: A 20 year experience, 177: 118-126.

9. Ensor, T. and Cooper, S. (2004). Overcoming barriers to health service access: influencing the demand side, 19(2): 69-79.

10. Gertler, P. and Van der Gaag, J. (1990). The willingness to pay for medical care: evidence from two developing countries.

11. Gopalan, S. S. and Durairaj, V. (2012). Addressing maternal healthcare through demand side financial incentives: experience of janani suraksha yojana program in india, 12(1): 1 .

12. Gupta, S. K., Pal, D. K., Tiwari, R., Garg, R. and Sarawagi, R. (2011). Assessment of Janani suraksha yojana (jsy) in jabalpur, madhya pradesh: knowledge, attitude and utilization pattern of beneficiaries: a descriptive study, 1(2): 06-11.

13. Gupta, S. K., Pal, D. K., Tiwari, R., Garg, R., Shrivastava, A. K., Sarawagi, R., Patil, R., Agarwal, L., Gupta, P. and Lahariya, C. (2012). Impact of janani suraksha yojana on institutional delivery rate and maternal morbidity and mortality: an observational study in Indi, 30(4): 464-471.

14. Ki-Moon, B. (2010). Global strategy for womens and childrens health,

15. Kingkaew, P., Werayingyong, P., Aye, S., Tin, N., Singh, A., Myint, P. and Teerawattananon, Y. (2016). An ex-ante economic evaluation of the maternal and child health voucher scheme as a decision-making tool in myanmar, 31(4): 482-492.
16. Koblinsky, M., Matthews, Z., Hussein, J., Mavalankar, D., Mridha, M. K., Anwar, I., Achadi, E., Adjei, S., Padmanabhan, P., van Lerberghe, W. et al. (2006). Going to scale with professional skilled care, The Lancet 368(9544): 1377-1386.

17. Kuwawenaruwa, A., Mtei, G., Baraka, J. and Tani, K. (2016). Implementing demand side targeting mechanisms for maternal and child health-experiences from national health insurance fund program in rungwe district, tanzania,

18. Lim, S. S., Dandona, L., Hoisington, J. A., James, S. L., Hogan, M. C. and Gakidou, E. (2010). India's janani suraksha yojana, a conditional cash transfer programme to increase births in health facilities: an impact evaluation, 2009-2023.

19. Modugu, H. R., Kumar, M., Kumar, A. and Millett, C. (2012). State and socio demographic group variation in out-of-pocket expenditure, borrowings and janani suraksha yojana (jsy) programme use for birth deliveries in india, 12(1): 1 .

20. MoHFW (2017) Annual Report 2016-2017: Ministry of Health and Family Welfare. Government of India, New

21. Delhi, India.

22. Nandi, A. and Laxminarayan, R. (2016). The unintended effects of cash transfers on fertility: evidence from the safe motherhood scheme in india, 29(2): 457-491.

23. Powell-Jackson, T., Mazumdar, S. and Mills, A. (2015). Financial incentives in health: New evidence from India's janani suraksha yojana, 43: 154-169.

24. Registrar General of India (2013). Special bulletin on maternal mortality in india 2010-12. Sample registration system.

25. Skiles, M., Curtis, S., Basinga, P., Angeles, G. and Thirumurthy, H. (2015). The effect of performance-based financing on illness, careseeking and treatment among children: An impact evaluation in rwanda, 15(1).

26. Tandon, A. (2016). Infant mortality rate reduced, India achieves MDG target, Technical report, The Tribune, New Delhi, India.

27. Thaddeus, S. and Maine, D. (1994). Too far to walk: maternal mortality in context, 38(8): 1091-1110.

28. Thomas EL., (2003). Propensity Scores: What Do They Do, How Should I Use Them, and- Why Should I Care?; Center for Health Care Research \& Policy Case, Western Reserve University.

29. United Nations International Children's Emergency Fund (UNICEF), (2017). UNICEF Data: Monitoring the Situation of Children and Women.

30. Van de Poel, E., Flores, G., Ir, P., Van Doorslaer, E. et al. (2014). Can vouchers deliver? an evaluation of subsidies for maternal health care in Cambodia 92(5): 331-339

31. World Heal Organisation (WHO) (2016). True magnitude of stillbirths and maternal and neonatal deaths underreported.

32. World Heal Organisation (WHO) (2017). Maternal, newborn, child and adolescent health.

33. Yang, L., Sun, L., Wen, L., Zhang, H., Li, C., et al. (2016). Financing strategies to improve essential public health equalization and its effects in china, 15(1): 1-12.

34. Yojana, J. S. (2006). Available from the official site of the Ministry of Health and Family Welfare, Government of India at: mohfw. nic.in/layout 09-06 